bridisierten Tetraeder-Eigenfunktionen zu beginnen. Da unser Molekül eine geschlossene $L$-Schale enthält, bleiben die Resultate hinsichtlich $Z_{0}, R$, $E, \chi$ dadurch unberührt. Jede „Tetraederfunktion" hat, abgesehen von der Richtung ihrer Symmetrieachse im Raum, die Form

$$
\varphi=\frac{1}{2} \psi_{2 s}+\frac{\sqrt{3}}{2} \psi_{2 p} .
$$

Damit erhält man für das Bindungsmoment den Wert

$$
\mu_{\mathrm{C}-\mathrm{H}}=0,44 \cdot 10^{-18} \text { e.s. E. Polarität } \mathrm{C}^{+} \mathrm{H}^{-},
$$

wenn man eine solche Funktion, mit zwei Elektronen besetzt, als für die entsprechende Bindung verantwortlich ansieht.

Der zuverlässigste experimentelle Wert ist 0,4 $\cdot 10^{-18}$ e.s.E. Der theoretische Wert, den C o u l* son mit der Methode der Molekül-Eigenfunktionen erhält, ist $0,40 \cdot 10^{-18}$ mit derselben Polarität wie oben angegeben.

\title{
Verdampfungsgleichgewichte von Mehrstoffgemischen II ${ }^{1}$
}

\author{
Von Rolf HaAse \\ Aus dem Physikalisch-chemischen Institut der Universität Marburg \\ (Z. Naturforschg. 2 a, 492-494 [1947]; eingegangen am 16. Juni 1947)
}

\begin{abstract}
Die Frage nach der Übertragbarkeit der K on ow a l ow schen Sätze von Zweistoffgemischen auf Mehrstoffgemische wird an Hand der Gib s schen Gleichungen für die Stabilität und Koexistenz von Phasen untersucht. Es ergeben sich wesentliche Einschränkungen, die für das Verhalten von Mehrstoffgemischen bei der Destillation und Rektifikation maßgebend sind.
\end{abstract}

1. Phasenstabilität und Phasen$\mathrm{k}$ oe $\mathrm{xistenz}$

E ine Phase aus $n$ Komponenten, charakterisiert durch die Molenbrüche $x_{1}, x_{2} \ldots x_{n-1}$, werde bei einer bestimmten Temperatur und einem bestimmten Druck auf ihre Stabilität untersucht in bezug auf Phasen in unmittelbarer Nachbarschaft, d.h. Phasen, deren Zusammensetzungen beliebig wenig von der Zusammensetzung der betrachteten Phase verschieden sind. Bildet man aus den zweiten Differentialquotienten der auf $1 \mathrm{Mol}$ Mischung bezogenen Gibbs schen Freien Energie (Freien Enthalpie) $G$ nach den Molenbrüchen folgende Determinante:

$$
D=\left|\begin{array}{ccc}
\frac{\partial^{2} G}{\partial x_{1}^{2}} & \frac{\partial^{2} G}{\partial x_{1} \partial x_{2}} \cdots \frac{\partial^{2} G}{\partial x_{1} \partial x_{n-1}} \\
\frac{\partial^{2} G}{\partial x_{2} \partial x_{1}} & \frac{\partial^{2} G}{\partial x_{2}^{2}} & \vdots \\
\vdots & & \frac{\partial^{2} G}{\partial x_{n-1} \partial x_{1}}
\end{array}\right|
$$

so ergibt sich nach $\mathrm{G}$ i b b s ${ }^{2,3}$, daß Stabilität einer Phase im obigen Sinne (also entweder absolute Stabilität oder Mefastabilität) vorliegt, wenn die
Determinante $D$ mit sämtlichen Hauptminoren positiv ist:

$$
D>, 0 \frac{\partial^{2} G}{\partial x_{1}^{2}}>0,\left|\begin{array}{cc}
\frac{\partial^{2} G}{\partial x_{1}^{2}} & \frac{\partial^{2} G}{\partial x_{1} \partial x_{2}} \\
\frac{\partial^{2} G}{\partial x_{2} \partial x_{1}} & \frac{\partial^{2} G}{\partial x_{2}^{2}}
\end{array}\right|>0 \text { usw. }
$$

Die Bedingung für die Koexistenz zweier Phasen bei Änderung der Intensitätsparameter (Druck $P$, Temperatur $T$, chemische Potentiale $\mu_{i}$ ) ergibt sich aus der Gibbs-Duhem schen Gleichung unter Beachtung der Tatsache, daß bei Gleichgewicht Druck, Temperatur und chemische Potentiale in beiden Phasen (unterschieden durch die Zahl der Striche) gleich sein müssen:

$\left(V^{\prime \prime}-V^{\prime}\right) d P-\left(S^{\prime \prime}-S^{\prime}\right) d T=\sum_{i=1}^{n}\left(x_{i}^{\prime \prime}-x_{i}^{\prime}\right) d \mu_{i}(2)$

( $V=$ Volumen, $S=$ Entropie, jeweils bezogen auf 1 Mol Mischung).

1 I. Mitt.: W. J os t, Z. Naturforschg. 1, 576 [1946].

2 The Scientific Papers of J. W. Gibbs I, 132 (Longmans, Green and Co. 1906).

3 A. St or onkin, Acta physicochim. URSS 13, 513 [1940]. 
Mit den Beziehungen

$$
\begin{aligned}
& \sum_{i}^{n-1} x_{i}=1-x_{n} \\
& \frac{\partial G}{d x_{i}}=\mu_{i}-\mu_{n}, \\
& d \frac{\partial G}{\partial x_{i}}=\frac{\partial V}{\partial x_{i}} d P-\frac{\partial S}{\partial x_{i}} d T+\sum_{k}^{n-1} \frac{\partial^{2} G}{\partial x_{i} \partial x_{k}} d x_{k} \\
& \text { erhält man : } \\
& \left\{V^{\prime \prime}-V^{\prime}-\sum_{i}^{n-1}\left(x_{i}^{\prime \prime}-x_{i}^{\prime}\right) \frac{\partial V}{\partial x_{i}}\right\} d P \\
& -\left\{S^{\prime \prime}-S^{\prime}-\sum_{\substack{i \\
n-1 \\
n-1}}^{n-1}\left(x_{i}^{\prime \prime}-x_{i}^{\prime}\right) \frac{\partial S}{\partial x_{i}}\right\} d T \\
& =\sum_{i} \sum_{k}\left(x_{i}^{\prime \prime}-x_{i}^{\prime}\right) \frac{\partial^{2} G}{\partial x_{i} \partial x_{k}} d x_{k} .
\end{aligned}
$$

Die nicht mit Strichen versehenen Differentialquotienten haben in beiden Phasen verschiedene Werte und beziehen sich wie $d x_{k}$ wahlweise auf eine der beiden Phasen, so daß man zwei Differentialgleichungen der Form (6) hat ${ }^{3}$.

\section{Die Sätze von Konowalow bei $\mathrm{Z}$ weist offgemischen}

Für binäre Mischungen $(n=2)$ folgt aus Gl. (6) :

$$
\begin{aligned}
& \left\{V^{\prime \prime}-V^{\prime}-\left(x^{\prime \prime}-x^{\prime}\right) \frac{\partial V}{\partial x}\right\} d P \\
& -\left\{S^{\prime \prime}-S^{\prime}-\left(x^{\prime \prime}-x^{\prime}\right) \frac{\partial S}{\partial x}\right\} d T \\
& =\left(x^{\prime \prime}-x^{\prime}\right) \frac{\partial^{2} G}{\partial x^{2}} d x ;
\end{aligned}
$$

$x=$ Molenbruch einer Komponente, $\frac{\partial V}{d x}, \frac{\partial S}{\partial x}$, $\frac{\partial^{2} G}{\partial x^{2}}$ und $d x$ beziehen sich wahlweise auf beide Phasen.

Es gilt ferner: $\frac{\partial^{2} G}{\partial x^{2}}>0$ für stabile (bzw. metastabile) Phasen.

Es werde nun das Gleichgewicht Flüssigkeit-Dampf betrachtet; die doppelt gestrichenen Größen mögen sich auf den Dampf, die einfach

4 J. P. K u en en, Theorie der Verdampfung und Verflüssigung von Gemischen, S. 50, 66 (Leipzig 1906). gestrichenen Größen auf die flüssige Phase beziehen. In diesem Falle sind die in geschweiften Klammern stehenden Ausdrücke in Gl. (6) und (7) stets positiv, falls man sich nicht in der Nähe kritischer Punkte befindet ${ }^{4}$.

Somit folgen aus Gl. (7) die drei Sätze von $\mathrm{K}$ on ow alow ${ }^{5}$, deren erster schon bei Gibbs ${ }^{6}$ $\mathrm{zu}$ finden ist:

1. Bei konst. Temperatur zeigt der Dampfdruck einer binären Mischung als Funktion der Zusammensetzung ein Extremum, wenn die Zusammensetzung beider Phasen gleich ist. (Es gilt auch die Umkehrung.)

2. Der Sampfdruck einer binären Mischung steigt bei konst. Temperatur durch Hinzufügen derjenigen Komponente, deren Konzentration im Dampf $\begin{gathered}\text { größer } \\ \text { kleiner }\end{gathered}$ als in der Flüssigkeit ist.

Daraus folgt:

2a. Bei kontinuierlicher Verdampfung (einfacher Destillation) $\begin{aligned} & \text { sinkt der Dampfdruck } \\ & \text { steigt der Siedepunkt }\end{aligned}$ einer binären flüissigen Mischung bei konst. Temperatur binären flüssigen Mischung bei konst. Tempertem Druck monoton. (Entsprechendes gilt für die Kondensation eines binären Dampfgemisches.)

3. Bei Veränderung von Druck oder Temperatur ändert sich die Zusammensetzung einer mit Dampf im Gleichgewicht befindlichen binären - Mischung in beiden Phasen im gleichen Sinne.

Aus diesen Sätzen ergibt sich das bekannte Verhalten binärer Gemische bei der Destillation und Rektifikation. Bei isobarer einfacher Destillation steigen Siedepunkt des Rückstandes und des Destillats monoton, bis Flüssigkeit und Dampf dieselbe Zusammensetzung haben. Bei der Rektifikation (ideales Trennvermögen der Kolonne vorausgesetzt) bleibt der Siedepunkt des Destillats konstant, bis der Rückstand eine konstant siedende Flüssigkeit (reiner Stoff oder azeotropes Gemisch) ist, um dann sprunghaft anzusteigen.

3. Gültigkeit der Sätze von Konowalow bei Mehrstoffgemischen

Aus Gl. (6) folgt:

Wenn $x^{\prime}=x_{i}^{\prime \prime}$, dann gilt:

für $T=$ const: $d P=0$,

für $P=$ const: $d T=0$,

5 D. Ko now al ow, Wied. Ann. Physik 14, 48 [1881].

6 J. W. Gi ib b s, Trans. Connect. Acad. 3 [1878]. 
da im Gleichgewicht Flüssigkeit-Dampf $V^{\prime} \neq V^{\prime \prime}$, $S^{\prime} \neq S^{\prime \prime}$ ist.

Diese Beziehung, dem ersten K o n ow a l ow schen Satze für binäre Mischungen entsprechend, ist schon bei $\mathrm{Gibbs}^{7}$ angedeutet. Den Beweis dafür, daß auch die Umkehrung gilt, findet man bei $\mathrm{Stor}$ onkin ${ }^{3}$.

Eine dem zweiten und dritten Satz von K o n o walow analoge Aussage kann für Mehrstoffgemische nicht gewonnen werden, solange man keine die Allgemeinheit einschränkenden Annahmen über die Funktion $G$ in Abhängigkeit von den $x_{i}$ macht.

Der zweite Satz kann hingegen in der eingeschränkten Form (2a) auch für Mehrstoffgemische aufrechterhalten werden, wie für Dreistoffgemische zuerst $\mathrm{S} \mathrm{ch}$ r e i n e m a k e r s ${ }^{8}$ zeigte und hier allgemein abgeleitet werden soll.

Läßt man $d n$ Mole einer aus insgesamt $n$ Molen bestehenden Flüssigkeit der Ausgangszusammensetzung $x_{1}^{\prime}+d x_{1}^{\prime} \ldots x_{k}^{\prime}+d x_{k}^{\prime} \ldots x_{n-1}^{\prime}+d x_{n-1}^{\prime}$ verdampfen, so habe die zurückbleibende Flüssigkeit die Zusammensetzung $x_{1}^{\prime} \ldots x_{k}^{\prime} \ldots x_{n-1}^{\prime}$; derGleichgewichtsdampf sei gegeben $\operatorname{durch} x_{1}^{\prime \prime} \ldots x_{k}^{\prime \prime} \ldots x_{n-1}^{\prime \prime}$. Der Satz von der Erhaltung der Masse fordert dann:

$$
d x_{k}^{\prime}=\frac{\left(x_{k}^{\prime \prime}-x_{k}^{\prime}\right) d n}{n}, \quad \frac{d x_{k}^{\prime}}{d x_{m}^{\prime}}=\frac{x_{k}^{\prime \prime}-x_{k}^{\prime}}{x_{m}^{\prime \prime}-x_{m}^{\prime}} .
$$

Die Indices $k$ und $m$ beziehen sich auf zwei beliebige Komponenten. Eine entsprechende Überlegung gilt für die Kondensation. Aus (6) und (8) ergibt sich:

$$
\begin{aligned}
& V_{0} d P-S_{0} d T \\
& =\frac{d x_{m}}{x_{m}^{\prime \prime}-x_{m}^{\prime}} \sum_{i}^{n-1} \sum_{i}^{n-1} \frac{\partial^{2} G}{\partial x_{i} \partial x_{k}}\left(x_{i}^{\prime \prime}-x_{i}^{\prime}\right)\left(x_{k}^{\prime \prime}-x_{k}^{\prime}\right),
\end{aligned}
$$

worin

$$
\begin{aligned}
& V_{0}=V^{\prime \prime}-V^{\prime}-\sum_{i}^{n-1}\left(x_{i}^{\prime \prime}-x_{i}^{\prime}\right) \frac{\partial V}{\partial x_{i}}>0, \\
& S_{0}=S^{\prime \prime}-S^{\prime}-\sum_{n-1}^{n}\left(x_{i}^{\prime \prime}-x_{i}^{\prime}\right) \frac{\partial S}{\partial x_{i}}>0 .
\end{aligned}
$$

Die Ausdrücke $\frac{\partial V}{\partial x_{i}}, \frac{\partial S}{\partial x_{i}}, \frac{\partial^{2} G}{\partial x_{i} \partial x_{k}}$ und $d x_{m}$ beziehen sich wieder wahlweise auf beide Phasen, je nachdem man den Verdampfungs- oder den Kondensationsvorgang betrachtet.

Da $\frac{\partial^{2} G}{\partial x_{i} \partial x_{k}}=\frac{\partial^{2} G}{\partial x_{k} \partial x_{i}}$, stellt die Doppelsumme in Gl. (9) wegen (1) eine positiv definite quadratische Form dar. Es folgt daher der Satz:

Verdampft bei konst. Temperatur ein Gemisch beliebig vieler Komponenten, so sinktder Dampfdruck monoton.

Ein sinngemäßer abgeänderter Satz gilt für die Kondensation eines Mehrstoffgemischs.

Aus diesen Befunden können wir für die Destillation von Mehrstoffgemischen allgemein folgendes aussagen:

Bei isobarer Destillation steigt der Siedepunkt des Rückstandes monoton. Der Siedepunkt des Destillats kann steigen oder fallen; es genügt nämlich schon die Ungültigkeit des zweiten oder dritten Satzes von K o n o w a low, um die Übertragung der oben genannten einfachen Gesetze für die Destillation und Rektifikation von binären Gemischen auf Mehrstoffgemische unmöglich zu machen.

In der Tat haben W. Re inders und C. H. de Minjer ${ }^{9}$ sowie R. H. Ewell und L. M. $\mathrm{Wel} \mathrm{ch}^{\mathbf{1 0}}$ bei der Rektifikation der ternären Gemische Benzol-Aceton-Chloroform und Methanol-Aceton-Chloroform ein zeitweiliges Sinken des Siedepunkts des Destillats beobachtet.

Die Berechnungen wurden auf Anregung von Hrn. Prof. W. Jost ausgeführt. Ich möchte ihm für das Interesse an der Arbeit herzlich danken.

7 Scient. Pap. J. W. Gi i b s I, 99 [1906]'.

${ }^{8}$ F. A. H. S chrein e m a ker s, Z. physik. Chem. 36, 271 [1901].

9 W. R e ind e r s u. C. H. d e Minjer, Recueil Trav. chim. Pays-Bas 59, 207, 369, 392 [1940].

${ }_{10}$ R. H. E w ell u. L. M. Wel ch, Ind. Engng. Chem. 37, 1224 [1945]. 